\title{
Analysis of the Proposed Nigerian 330KV Integrated Network using ETAP 12.6 software
}

\author{
${ }^{1}$ Ogbikaya S.ogbikaya.stephen@edouniversity.edu.ng \\ ${ }^{2}$ Ike S.sam.ike@uniben.edu \\ ${ }^{3}$ Evbogbai M. J. E. evbogbaiedekin@edouniversity.edu.ng \\ ${ }^{1 \& 3}$ Department of Electrical and Electronic Engineering, Edo University Iyamho, Nigeria. \\ ${ }^{2}$ Department of Electrical and Electronic Engineering, University of Benin, Nigeria. \\ All correspondence should be directed to Engr Ogbikaya S. (ogbikaya.stephen@edouniversity.edu.ng) \\ DOI: 10.31364/SCIRJ/v7.i1.2019.P0119XX \\ http://dx.doi.org/10.31364/SCIRJ/v7.i1.2019.P0119XX
}

\begin{abstract}
The analysis of the impact of integrating the power generated from Generation Company of Nigeria (GENCO), Nigeria Independent Power Project (NIPP) and the Independent Power Producers (IPP) into the existing 330kV transmission network is presented in this work. The analysis is carried out using ETAP 12.6 and MATLAB software for the existing and the proposed 330kV grid network. The result indicates that with the proposed integrated $330 \mathrm{kV}$ network, the power losses on each transmission lines reduced by $0.48 \mathrm{MWwhich}$ indicates $46 \%$ improvement on the existing $330 \mathrm{kV}$ transmission network and the voltage profile on each bus of the entire network improves from $(0.66-1.13)$ per unit to $(0.94-1.50)$ per unit. The result reveals that the integration of the proposed $330 \mathrm{kV}$ network, electricity supply in the national grid will be stable, reliable and adequate for sustainable development.
\end{abstract}

Keywords: 330kV, Networks, Integrated, Power Generation, ETAP 12.6, MATLAB.

\section{INTRODUCTION}

Electricity plays an indispensable role in the socio-economic and technological development of every nation, but Nigeria is faced with acute electricity challenges, thereby hindering her development in spite of the availability of vast national resources for power generation. Presently the country is faced with the challenges of providing sustainable, stable, adequate, reliable, efficient and economical electricity supply to residential, commercial and industrial consumers. This situation has adversely affected the socioeconomic and technological life of the citizenry. Onahaebi (2007) stated in his study of Reduction of the high technical power losses associated with the Nigerian 330KV Transmission Network using Power World Simulator (PWS) that the existing network cannot cope with contingencies leading to very low performance and even blackout at emergencies. He proposed modifications to the $330 \mathrm{KV}$ transmission network incorporating additional lines to form more loops with minimal compensation meets the acceptable limit of $\pm 5 \%$. It also reduces the energy losses from 337.5 - $189.9 \mathrm{MWH}$, representing $45 \%$ improvement over the existing network. Omorogiuwa and Ike (2014) in their work reported that Nigeria power system is faced with problems of inadequate power generation and transmission lines, resulting in the overloading and stressing of the network beyond their thermal limit because of the increasing load demand. Shaikh, Jain, Kotnala and Agarwal (2012), reported that the main factor which causes unacceptable voltage profile is the inability of the distribution system to meet the demand for reactive power. Agarwal concluded that for voltage stability, the maximum permissible loading limits must not be exceeded in the operation of the power system. Sambo, Garba, Zarma and Gaji, (2012) in their work stated that adequate power supply is an unavoidable prerequisite to any nation's development. To provide adequate power to ensure that Nigeria is among the industrialized nations, three critical activities must be effectively achieved, they are: adequate power must be generated, the power generated must effectively be transmitted to all part of the country and finally the power transmitted must be effectively distributed to the consumers. Since development and population growth in any country are 
highly dynamic, these three activities must also be carefully addressed in a dynamic, creative and logical manner. The epileptic nature of electricity generation in Nigeria associated with the existing 330KV network has become unbearable to most Nigerians, especially in the big cities. It has posed a constant threat to the growth of the country's economy. The existing 330KV network has series of drawback ranging from inadequate generation, weak and fragile transmission lines that are not robust enough to wheel out the generated power in the network. The high load demand on the network makes the network prone to voltage instability which causes voltage limit violation in some of the buses that may lead to voltage collapse in the entire network. The voltage problems associated with this network are caused by long distance power transmission (ie load centres far away from generating stations) and overloading of the transmission line (ie transmission lines carrying load beyond their available transfer capacity). The high load demand on the network result to system insecurity and several voltage collapses on the network, which makes it necessary for power system integration.

In this work, the analysis of the impact of integrating the power generated by Generation Company of Nigeria (GENCO), Nigeria Independent Power Project (NIPP) and the Independent Power Producers (IPP) into the existing 330KV transmission network using the ETAP 12.6 software is presented.

\section{MATERIALS AND METHOD}

The materials used in the analysis of this work are ETAP 12.6 software, the Existing 330KV grid network, the Proposed 330KV grid network and MATLAB software.

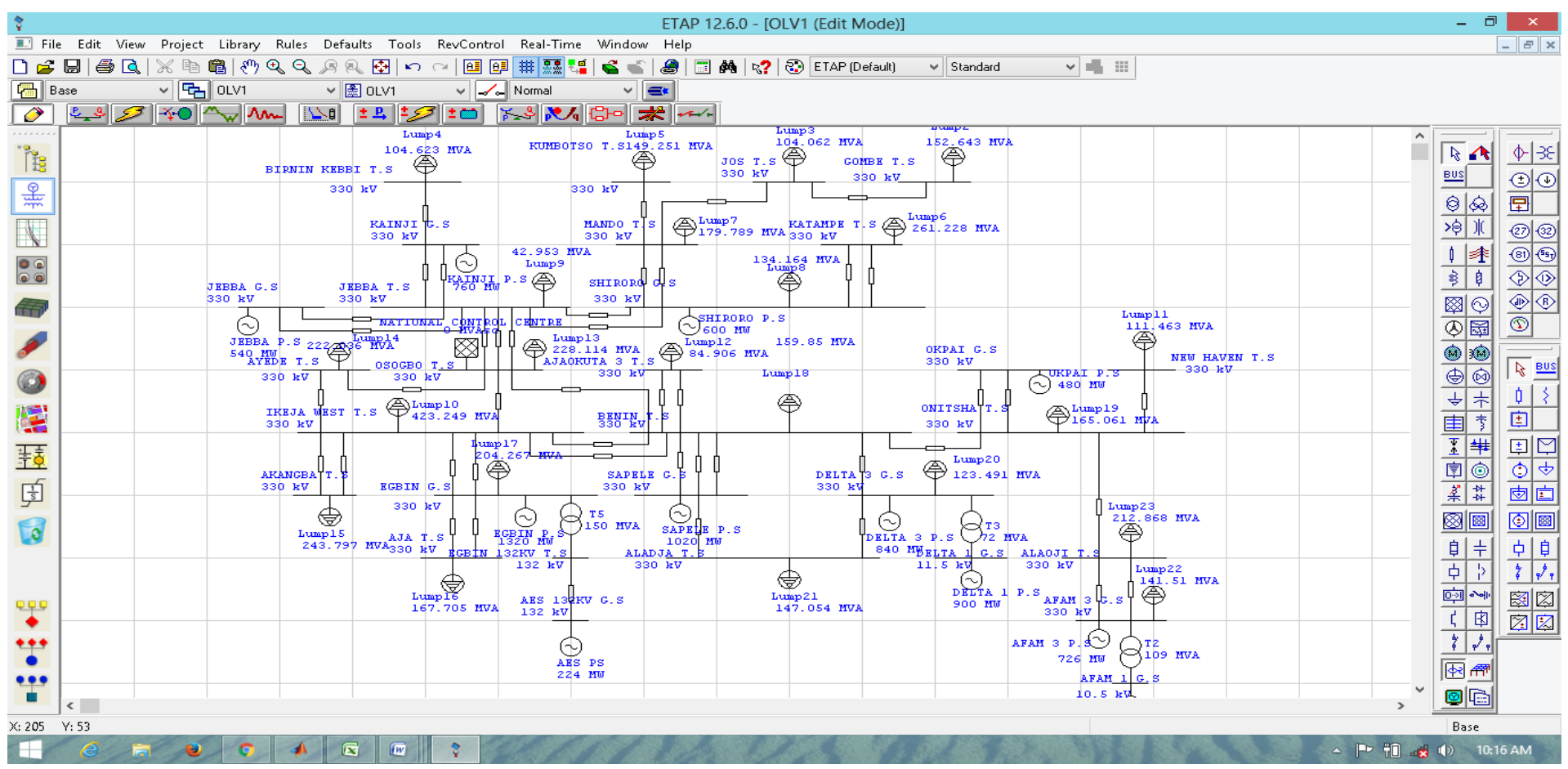

Figure 1: Model of Existing 330KV Network using ETAP 12.6 Software

The research was achieved through modelling and simulation involving the following approaches:

i. Model development of the existing 330KV Network and proposed integrated 330KV Network using ETAP 12.6 as shown in figure 1 and figure 3. 
ii. Simulation (run mode) of the developed existing 330KV Network and proposed integrated 330KV Network model using ETAP 12.6 with Newton Raphson Iteration Algorithm having a precision of 0.0001, as shown in figure 2 and figure 4 .

iii. The performance of the existing 330KV Network and proposed integrated 330KV Network model was achieved by analyzing simulation result using MATLAB.

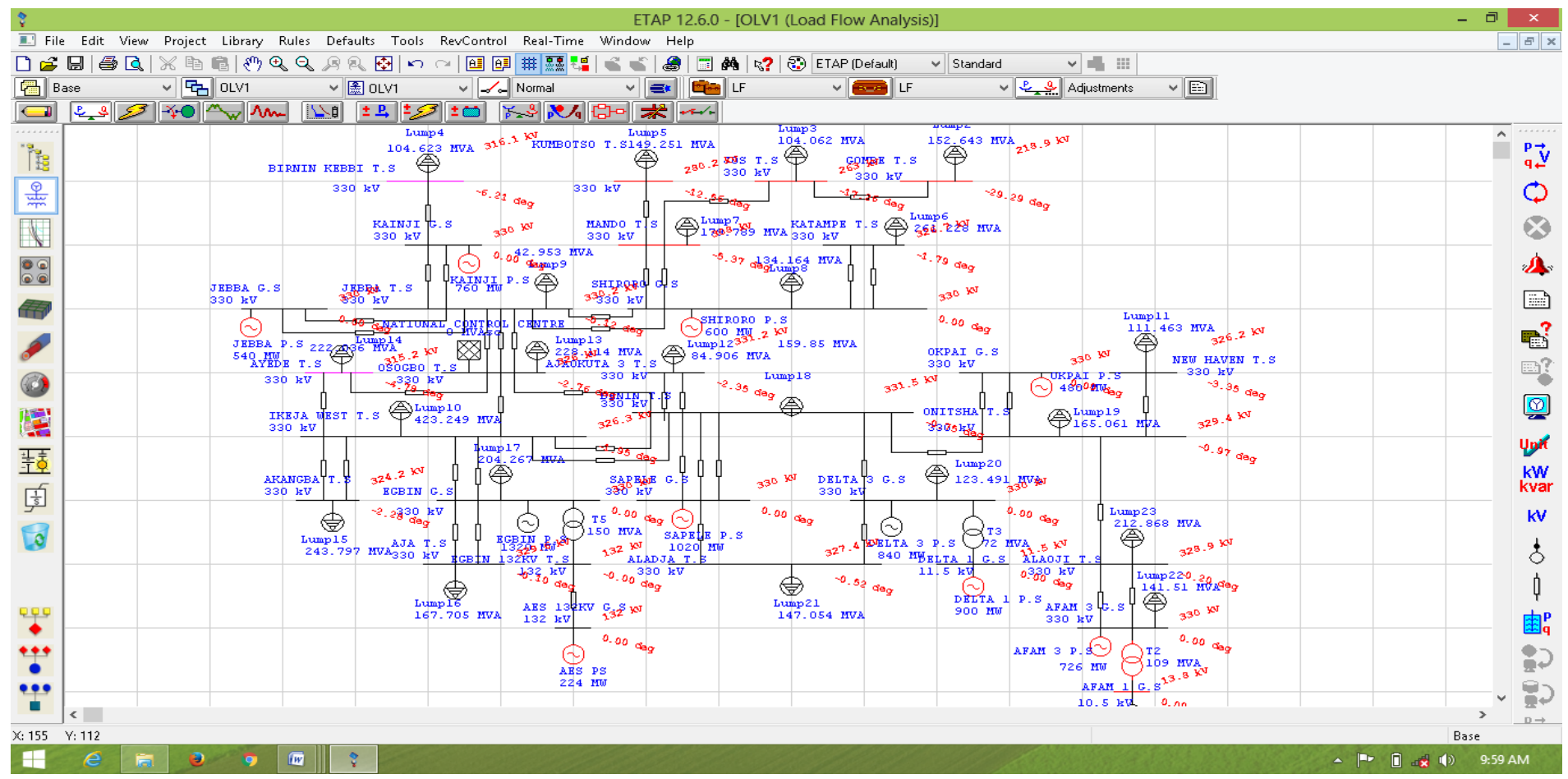

Figure 2: Simulation (Run Mode) of Existing 330KV Network using ETAP 12.6 Software

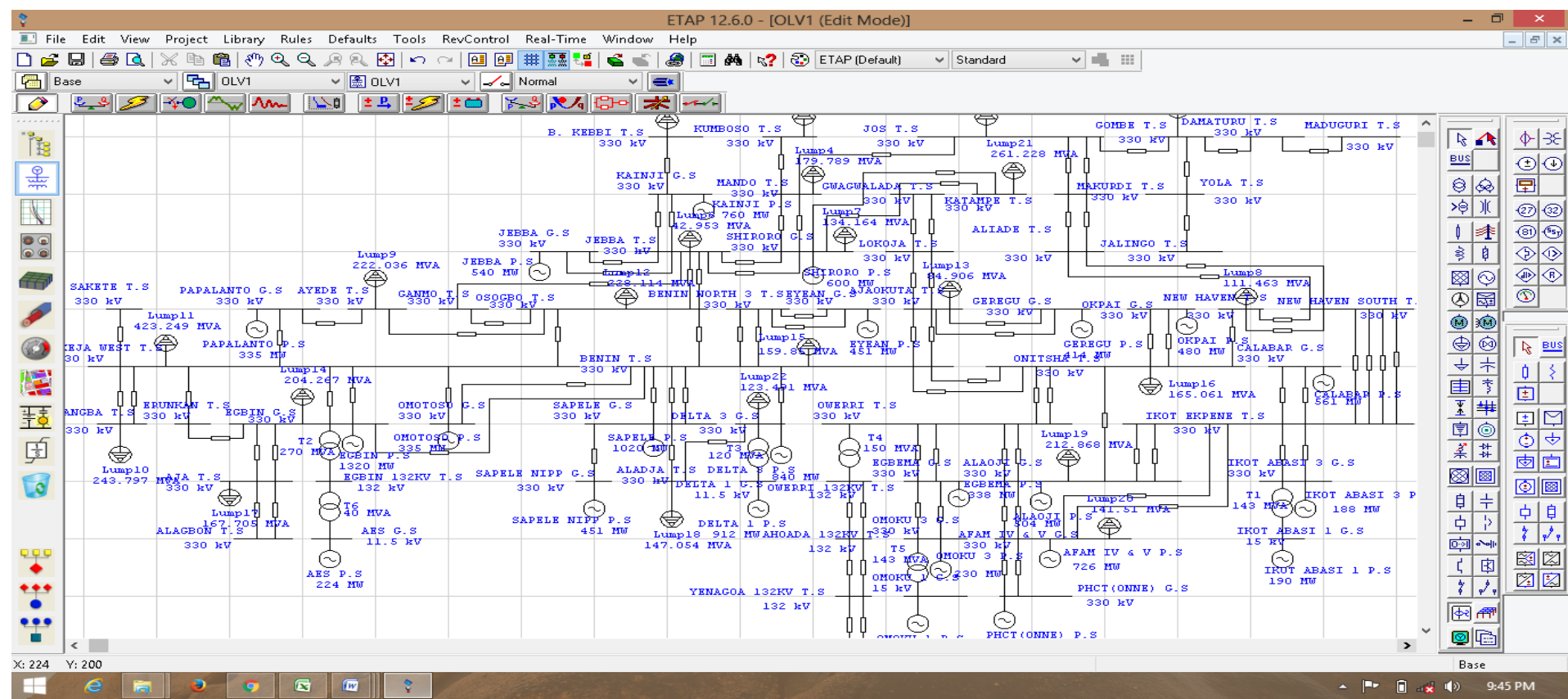

Figure 3: Model of Proposed Integrated 330KV Network using ETAP 12.6 Software 


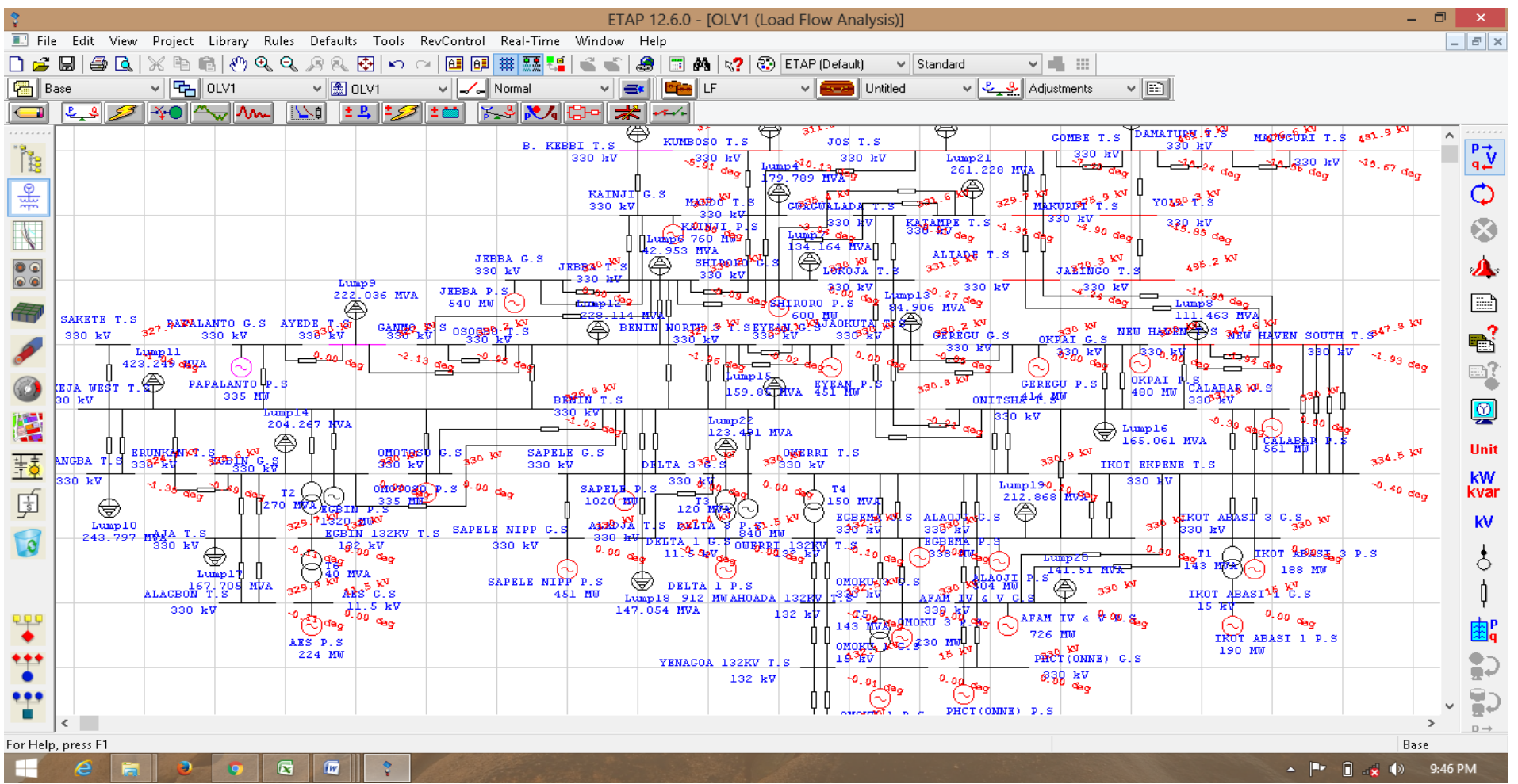

Figure 4: Simulation (Run mode) of Proposed Integrated 330KV Network using ETAP 12.6 Software

\section{RESULT AND DISCUSSION}

The result obtained from the simulation indicate that the real and reactive power losses in the existing 330KV network are 49.72MW and -1525.49 MVar respectively, with the following transmission stations having their operating voltages below the acceptable voltage limit of $\pm 5 \%$; Gombe T.S 218.91KV, Jos T.S 263.05KV, Kumbotso T.S 280.25KV and Mando T.S 308KV as shown in table 1.

Table 1: Performance of Existing 330KV Network with ETAP 12.6

\begin{tabular}{|c|l|c|c|c|c|l|}
\hline $\begin{array}{c}\text { Bus } \\
\text { No }\end{array}$ & \multicolumn{1}{|c|}{ Bus ID } & Nominal kV & Voltage & Voltage PU & $\%$ Voltage Drop & \multicolumn{1}{|c|}{ Remark } \\
\hline 1 & AFAM 3 G.S & 330 & 330 & 1 & 0 & Acceptable Voltage limit \\
\hline 2 & AJA T.S & 330 & 329.59 & 0.99 & 0.12 & Acceptable Voltage limit \\
\hline 3 & AJAOKUTA 3 T.S & 330 & 331.16 & 1 & -0.35 & Acceptable Voltage limit \\
\hline 4 & AKANGBA T.S & 330 & 324.17 & 0.98 & 1.77 & Acceptable Voltage limit \\
\hline 5 & ALADJA T.S & 330 & 327.44 & 0.99 & 0.78 & Acceptable Voltage limit \\
\hline 6 & ALAOJI T.S & 330 & 328.89 & 0.99 & 0.34 & Acceptable Voltage limit \\
\hline 7 & AYEDE T.S & 330 & 315.21 & 0.96 & 4.48 & Acceptable Voltage limit \\
\hline 8 & BENIN T.S & 330 & 331.46 & 1 & -0.44 & Acceptable Voltage limit \\
\hline 9 & BIRNIN KEBBI & 330 & 316.14 & 0.96 & 4.2 & Acceptable Voltage limit \\
\hline
\end{tabular}




\begin{tabular}{|l|l|c|c|c|c|l|}
10 & 330 & 330 & 1 & 0 & Acceptable Voltage limit \\
\hline 11 & EGBIN G.S & 330 & 330 & 1 & 0 & Acceptable Voltage limit \\
\hline 12 & GOMBE T.S & 330 & 218.91 & 0.66 & 33.67 & $\begin{array}{l}\text { Below acceptable Volt. } \\
\text { Limit }\end{array}$ \\
\hline 13 & IKEJA WEST T.S & 330 & 326.31 & 0.99 & 1.12 & Acceptable Voltage limit \\
\hline 14 & JEBBA G.S & 330 & 330 & 1 & 1 & Acceptable Voltage limit \\
\hline 15 & JEBBA T.S & 330 & 330.16 & 1 & 1 & Acceptable Voltage limit \\
\hline 16 & JOS T.S & 330 & 263.05 & 0.8 & 20.29 & $\begin{array}{l}\text { Lelow acceptable Volt. } \\
\text { Limit }\end{array}$ \\
\hline 17 & KAINJI G.S & 330 & 330 & 1 & 0 & Acceptable Voltage limit \\
\hline 18 & KATAMPE T.S & 330 & 326.68 & 0.99 & 1.01 & Acceptable Voltage limit \\
\hline 19 & KUMBOTSO T.S & 330 & 280.25 & 0.85 & 15.08 & $\begin{array}{l}\text { Below acceptable Volt. } \\
\text { Limit }\end{array}$ \\
\hline 20 & MANDO T.S & 330 & 308 & 0.93 & 6.67 & $\begin{array}{l}\text { Below acceptable Volt. } \\
\text { Limit }\end{array}$ \\
\hline 21 & NEW HAVEN T.S & 330 & 326.16 & 0.99 & 1.16 & Acceptable Voltage limit \\
\hline 22 & OKPAI G.S & 330 & 330 & 1 & 0 & Acceptable Voltage limit \\
\hline 23 & ONITSHA T.S & 330 & 329.38 & 1 & 0 & Acceptable Voltage limit \\
\hline 24 & OSOGBO T.S & 330 & 325.96 & 0.99 & 1.22 & Acceptable Voltage limit \\
\hline 25 & SAPELE G.S & 330 & 330 & 1 & 0 & Acceptable Voltage limit \\
\hline 26 & SHIRORO G.S & 330 & 330 & 1 & 0 & Acceptable Voltage limit \\
\hline
\end{tabular}

The result in Table 1 indicates that four (4) out of the thirty (30) buses of the existing 330KV network are out of acceptable voltage limit which is $\pm 5 \%$ of nominal voltage, this implies that these transmission stations will go out one after the other thus causing system instability in the entire network that may result to system collapse. This is the major cause of system collapses that occur in the network. The graph of \% Voltage Drop versus Bus number of the existing 330KV network using MATLAB 7.5.0 is shown in figure 5. The graph clearly shows the four buses that are out of acceptable limit.

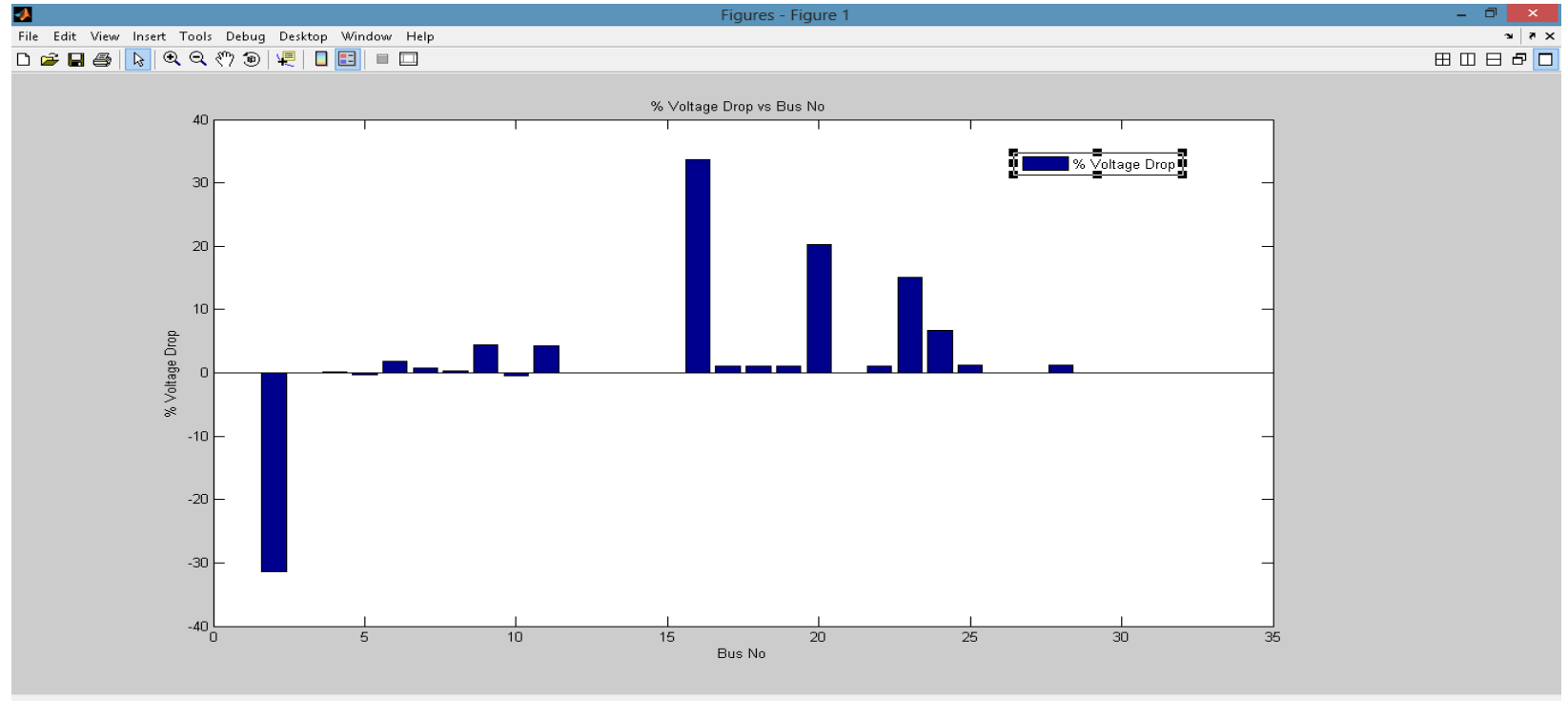




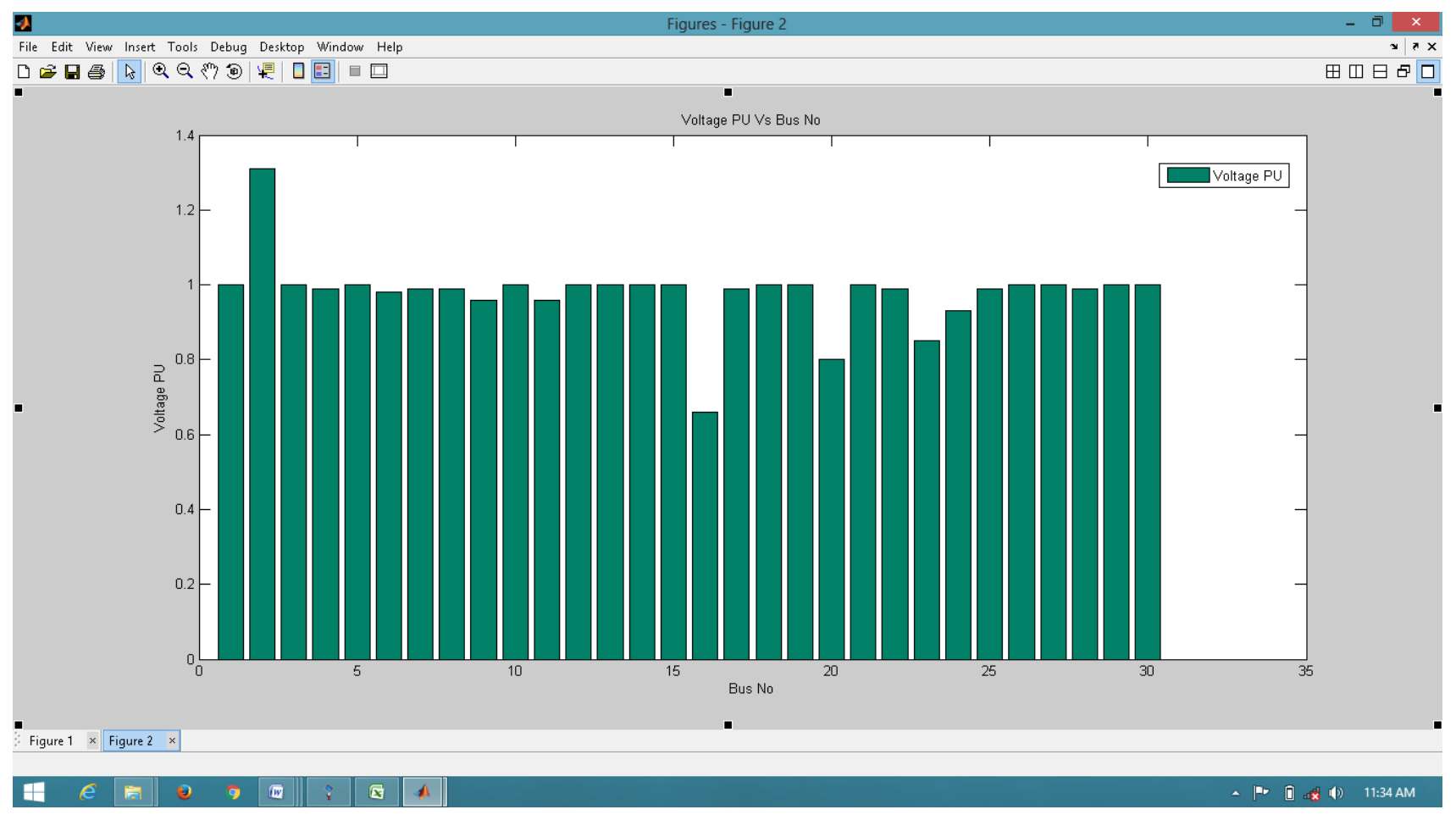

Figure 6: Voltage per unit Vs Bus No of Existing 330KV Network

Similarly, plotting the graph of Voltage per unit versus Bus number of the existing 330KV network using MATLAB 7.5.0 as shown in figure 6 was obtained.

This graph also clearly indicate the buses that were out of acceptable per unit value of (0.95-1.05) per unit which are Gombe T.S 218.91KV, Jos T.S 263.05KV, Kumbotso T.S 280.25KV and Mando T.S 308KV. This analysis reveals that the Existing $330 \mathrm{KVnetwork}$ has to be improved upon by the integration of the NIPP and the IPP generated power. 
Proposed Integrated 330KV Network: The Proposed Integrated 330KV transmission network consists of twenty-four (24) generating stations with an installed capacity of 12,510MW. It has sixty-one (61) buses with one hundred and eleven (111) branches with a transmission route length of 7,250km, having four national control centres at Osogbo, Benin, Ajaokuta and Onitsha. The following results were obtained from the load flow analysis of the Proposed Integrated 330KV network. The results obtained from the load flow analysis of the Proposed Integrated 330KV network is given in table 2.

Table 2: Performance of proposed integrated 330KV network with ETAP 12.6

\begin{tabular}{|c|c|c|c|c|c|c|}
\hline $\begin{array}{l}\text { Bus } \\
\text { No }\end{array}$ & Bus ID & $\begin{array}{c}\text { Nominal } \\
\mathrm{kV}\end{array}$ & Voltage & $\begin{array}{c}\text { Voltage } \\
\text { PU }\end{array}$ & $\begin{array}{c}\% \\
\text { Voltage } \\
\text { Drop }\end{array}$ & Remark \\
\hline 1 & AES G.S & 11.5 & 11.50 & 1.00 & 0.00 & $\begin{array}{l}\text { Acceptable Voltage } \\
\text { limit }\end{array}$ \\
\hline 2 & $\begin{array}{l}\text { AFAM IV \& V } \\
\text { G.S }\end{array}$ & 330.0 & 330.00 & 1.00 & 0.00 & $\begin{array}{l}\text { Acceptable Voltage } \\
\text { limit }\end{array}$ \\
\hline 3 & $\begin{array}{l}\text { AHOADA 132KV } \\
\text { T.S }\end{array}$ & 132.0 & 132.46 & 1.00 & -0.35 & $\begin{array}{l}\text { Acceptable Voltage } \\
\text { limit }\end{array}$ \\
\hline 4 & AJA T.S & 330.0 & 329.73 & 1.00 & 0.08 & $\begin{array}{l}\text { Acceptable Voltage } \\
\text { limit }\end{array}$ \\
\hline 5 & AJAOKUTA T.S & 330.0 & 330.21 & 1.00 & -0.06 & $\begin{array}{l}\text { Acceptable Voltage } \\
\text { limit }\end{array}$ \\
\hline 6 & AKANGBA T.S & 330.0 & 324.69 & 0.98 & 1.61 & $\begin{array}{l}\text { Acceptable Voltage } \\
\text { limit }\end{array}$ \\
\hline 7 & ALADJA T.S & 330.0 & 327.44 & 0.99 & 0.78 & $\begin{array}{l}\text { Acceptable Voltage } \\
\text { limit }\end{array}$ \\
\hline 8 & ALAGBON T.S & 330.0 & 329.85 & 1.00 & 0.05 & $\begin{array}{l}\text { Acceptable Voltage } \\
\text { limit }\end{array}$ \\
\hline 9 & ALAOJI G.S & 330.0 & 330.00 & 1.00 & 0.00 & $\begin{array}{l}\text { Acceptable Voltage } \\
\text { limit }\end{array}$ \\
\hline 10 & ALIADE T.S & 330.0 & 370.31 & 1.12 & -12.22 & $\begin{array}{l}\text { Above acceptable Volt. } \\
\text { Limit }\end{array}$ \\
\hline 11 & AYEDE T.S & 330.0 & 322.02 & 0.98 & 2.42 & $\begin{array}{l}\text { Acceptable Voltage } \\
\text { limit }\end{array}$ \\
\hline 12 & B. KEBBI T.S & 330.0 & 316.66 & 0.96 & 4.04 & $\begin{array}{l}\text { Acceptable Voltage } \\
\text { limit }\end{array}$ \\
\hline 13 & $\begin{array}{l}\text { BENIN NORTH } 3 \\
\text { T.S }\end{array}$ & 330.0 & 330.09 & 1.00 & -0.03 & $\begin{array}{l}\text { Acceptable Voltage } \\
\text { limit }\end{array}$ \\
\hline 14 & BENIN T.S & 330.0 & 330.77 & 1.00 & -0.23 & $\begin{array}{l}\text { Acceptable Voltage } \\
\text { limit }\end{array}$ \\
\hline
\end{tabular}




\begin{tabular}{|c|c|c|c|c|c|c|}
\hline 15 & CALABAR G.S & 330.0 & 330.00 & 1.00 & 0.00 & $\begin{array}{l}\text { Acceptable Voltage } \\
\text { limit }\end{array}$ \\
\hline 16 & DAMATURU T.S & 330.0 & 476.61 & 1.45 & -44.43 & $\begin{array}{l}\text { Above acceptable Volt. } \\
\text { Limit }\end{array}$ \\
\hline 17 & DELTA 1 G.S & 11.5 & 11.50 & 1.00 & 0.00 & $\begin{array}{l}\text { Acceptable Voltage } \\
\text { limit }\end{array}$ \\
\hline 18 & DELTA 3 G.S & 330.0 & 330.00 & 1.00 & 0.00 & $\begin{array}{l}\text { Acceptable Voltage } \\
\text { limit }\end{array}$ \\
\hline 19 & EGBEMA G.S & 330.0 & 330.00 & 1.00 & 0.00 & $\begin{array}{l}\text { Acceptable Voltage } \\
\text { limit }\end{array}$ \\
\hline 20 & $\begin{array}{l}\text { EGBIN 132KV } \\
\text { T.S }\end{array}$ & 132.0 & 132.00 & 1.00 & 0.00 & $\begin{array}{l}\text { Acceptable Voltage } \\
\text { limit }\end{array}$ \\
\hline 21 & EGBIN G.S & 330.0 & 330.00 & 1.00 & 0.00 & $\begin{array}{l}\text { Acceptable Voltage } \\
\text { limit }\end{array}$ \\
\hline 22 & ERUNKAN T.S & 330.0 & 328.63 & 0.99 & 0.42 & $\begin{array}{l}\text { Acceptable Voltage } \\
\text { limit }\end{array}$ \\
\hline 23 & EYEAN G.S & 330.0 & 330.00 & 1.00 & 0.00 & $\begin{array}{l}\text { Acceptable Voltage } \\
\text { limit }\end{array}$ \\
\hline 24 & GANMO T.S & 330.0 & 330.24 & 1.00 & -0.07 & $\begin{array}{l}\text { Acceptable Voltage } \\
\text { limit }\end{array}$ \\
\hline 25 & $\begin{array}{l}\text { GBARAN-UBIE } \\
\text { G.S }\end{array}$ & 132.0 & 132.00 & 1.00 & 0.00 & $\begin{array}{l}\text { Acceptable Voltage } \\
\text { limit }\end{array}$ \\
\hline 26 & GEREGU G.S & 330.0 & 330.00 & 1.00 & 0.00 & $\begin{array}{l}\text { Acceptable Voltage } \\
\text { limit }\end{array}$ \\
\hline 27 & GOMBE T.S & 330.0 & 461.61 & 1.40 & -39.88 & $\begin{array}{l}\text { Above acceptable Volt. } \\
\text { Limit }\end{array}$ \\
\hline 28 & $\begin{array}{l}\text { GWAGWALADA } \\
\text { T.S }\end{array}$ & 330.0 & 331.56 & 1.01 & -0.47 & $\begin{array}{l}\text { Acceptable Voltage } \\
\text { limit }\end{array}$ \\
\hline 29 & IKEJA WEST T.S & 330.0 & 326.83 & 0.99 & 0.96 & $\begin{array}{l}\text { Acceptable Voltage } \\
\text { limit }\end{array}$ \\
\hline 30 & $\begin{array}{l}\text { IKOT ABASI } 1 \\
\text { G.S }\end{array}$ & 15.0 & 15.00 & 1.00 & 0.00 & $\begin{array}{l}\text { Acceptable Voltage } \\
\text { limit }\end{array}$ \\
\hline 31 & $\begin{array}{l}\text { IKOT ABASI } 3 \\
\text { G.S }\end{array}$ & 330.0 & 330.00 & 1.00 & 0.00 & $\begin{array}{l}\text { Acceptable Voltage } \\
\text { limit }\end{array}$ \\
\hline 32 & $\begin{array}{l}\text { IKOT EKPENE } \\
\text { T.S }\end{array}$ & 330.0 & 334.47 & 1.01 & -1.35 & $\begin{array}{l}\text { Acceptable Voltage } \\
\text { limit }\end{array}$ \\
\hline 33 & JALINGO T.S & 330.0 & 495.16 & 1.50 & -50.05 & $\begin{array}{l}\text { Above acceptable Volt. } \\
\text { Limit }\end{array}$ \\
\hline
\end{tabular}




\begin{tabular}{|c|c|c|c|c|c|c|}
\hline 34 & JEBBA G.S & 330.0 & 330.00 & 1.00 & 0.00 & $\begin{array}{l}\text { Acceptable Voltage } \\
\text { limit }\end{array}$ \\
\hline 35 & JEBBA T.S & 330.0 & 330.23 & 1.00 & -0.07 & $\begin{array}{l}\text { Acceptable Voltage } \\
\text { limit }\end{array}$ \\
\hline 36 & JOS T.S & 330.0 & 388.33 & 1.18 & -17.68 & $\begin{array}{l}\text { Above acceptable Volt. } \\
\text { Limit }\end{array}$ \\
\hline 37 & KAINJI G.S & 330.0 & 330.00 & 1.00 & 0.00 & $\begin{array}{l}\text { Acceptable Voltage } \\
\text { limit }\end{array}$ \\
\hline 38 & KATAMPE T.S & 330.0 & 329.69 & 1.00 & 0.09 & $\begin{array}{l}\text { Acceptable Voltage } \\
\text { limit }\end{array}$ \\
\hline 39 & KUMBOSO T.S & 330.0 & 311.29 & 0.94 & 5.67 & $\begin{array}{l}\text { Acceptable Voltage } \\
\text { limit }\end{array}$ \\
\hline 40 & LOKOJA T.S & 330.0 & 331.48 & 1.00 & -0.45 & $\begin{array}{l}\text { Acceptable Voltage } \\
\text { limit }\end{array}$ \\
\hline 41 & MADUGURI T.S & 330.0 & 481.91 & 1.46 & -46.03 & $\begin{array}{l}\text { Above acceptable Volt. } \\
\text { Limit }\end{array}$ \\
\hline 42 & MAKURDI T.S & 330.0 & 375.85 & 1.14 & -13.89 & $\begin{array}{l}\text { Above acceptable Volt. } \\
\text { Limit }\end{array}$ \\
\hline 43 & MANDO T.S & 330.0 & 335.39 & 1.02 & -1.63 & $\begin{array}{l}\text { Acceptable Voltage } \\
\text { limit }\end{array}$ \\
\hline 44 & $\begin{array}{l}\text { NEW HAVEN } \\
\text { SOUTH T.S }\end{array}$ & 330.0 & 347.82 & 1.05 & -5.40 & $\begin{array}{l}\text { Above acceptable Volt. } \\
\text { Limit }\end{array}$ \\
\hline 45 & $\begin{array}{l}\text { NEW HAVEN } \\
\text { T.S }\end{array}$ & 330.0 & 347.60 & 1.05 & -5.33 & $\begin{array}{l}\text { Above acceptable Volt. } \\
\text { Limit }\end{array}$ \\
\hline 46 & OKPAI G.S & 330.0 & 330.00 & 1.00 & 0.00 & $\begin{array}{l}\text { Acceptable Voltage } \\
\text { limit }\end{array}$ \\
\hline 47 & OMOKU 1 G.S & 15.0 & 15.00 & 1.00 & 0.00 & $\begin{array}{l}\text { Acceptable Voltage } \\
\text { limit }\end{array}$ \\
\hline 48 & OMOKU 3 G.S & 330.0 & 330.00 & 1.00 & 0.00 & $\begin{array}{l}\text { Acceptable Voltage } \\
\text { limit }\end{array}$ \\
\hline 49 & OMOTOSO G.S & 330.0 & 330.00 & 1.00 & 0.00 & $\begin{array}{l}\text { Acceptable Voltage } \\
\text { limit }\end{array}$ \\
\hline 50 & ONITSHA T.S & 330.0 & 331.90 & 1.00 & -0.58 & $\begin{array}{l}\text { Acceptable Voltage } \\
\text { limit }\end{array}$ \\
\hline 51 & OSOGBO T.S & 330.0 & 327.83 & 0.99 & 0.66 & $\begin{array}{l}\text { Acceptable Voltage } \\
\text { limit }\end{array}$ \\
\hline 52 & $\begin{array}{l}\text { OWERRI 132KV } \\
\text { T.S }\end{array}$ & 132.0 & 132.49 & 1.00 & -0.37 & $\begin{array}{l}\text { Acceptable Voltage } \\
\text { limit }\end{array}$ \\
\hline
\end{tabular}




\begin{tabular}{|c|l|c|c|c|c|l|}
53 & OWERRI T.S & 330.0 & 330.89 & 1.00 & -0.27 & $\begin{array}{l}\text { Acceptable Voltage } \\
\text { limit }\end{array}$ \\
\hline 54 & $\begin{array}{l}\text { PAPALANTO } \\
\text { G.S }\end{array}$ & 330.0 & 330.00 & 1.00 & 0.00 & $\begin{array}{l}\text { Acceptable Voltage } \\
\text { limit }\end{array}$ \\
\hline 55 & $\begin{array}{l}\text { PHCT(ONNE) } \\
\text { G.S }\end{array}$ & 330.0 & 330.00 & 1.00 & 0.00 & $\begin{array}{l}\text { Acceptable Voltage } \\
\text { limit }\end{array}$ \\
\hline 56 & SAKETE T.S & 330.0 & 327.73 & 0.99 & 0.69 & $\begin{array}{l}\text { Acceptable Voltage } \\
\text { limit }\end{array}$ \\
\hline 57 & SAPELE G.S & 330.0 & 330.00 & 1.00 & 0.00 & $\begin{array}{l}\text { Acceptable Voltage } \\
\text { limit }\end{array}$ \\
\hline 58 & $\begin{array}{l}\text { SAPELE NIPP } \\
\text { G.S }\end{array}$ & 330.0 & 330.00 & 1.00 & 0.00 & $\begin{array}{l}\text { Acceptable Voltage } \\
\text { limit }\end{array}$ \\
\hline 59 & SHIRORO G.S & 330.0 & 330.00 & 1.00 & 0.00 & $\begin{array}{l}\text { Acceptable Voltage } \\
\text { limit }\end{array}$ \\
\hline 60 & $\begin{array}{l}\text { YENAGOA } \\
132 K V \text { T.S }\end{array}$ & 132.0 & 132.06 & 1.00 & -0.05 & $\begin{array}{l}\text { Acceptable Voltage } \\
\text { limit }\end{array}$ \\
\hline 61 & YOLA T.S & 330.0 & 490.33 & 1.48 & -48.58 & $\begin{array}{l}\text { Above acceptable Volt. } \\
\text { Limit }\end{array}$ \\
\hline
\end{tabular}

The buses with the voltage level above acceptable voltage limit are as a result of the low load demand on the transmission station hence a more stable and reliable network will be obtained by this integration. The results obtained in table 2 was analyzed graphically using MATLAB 7.5.0. Software, hence plotting the graph of \%Voltage Drop versus Bus number for the Proposed Integrated 330kV network, figure 7 was obtained. 


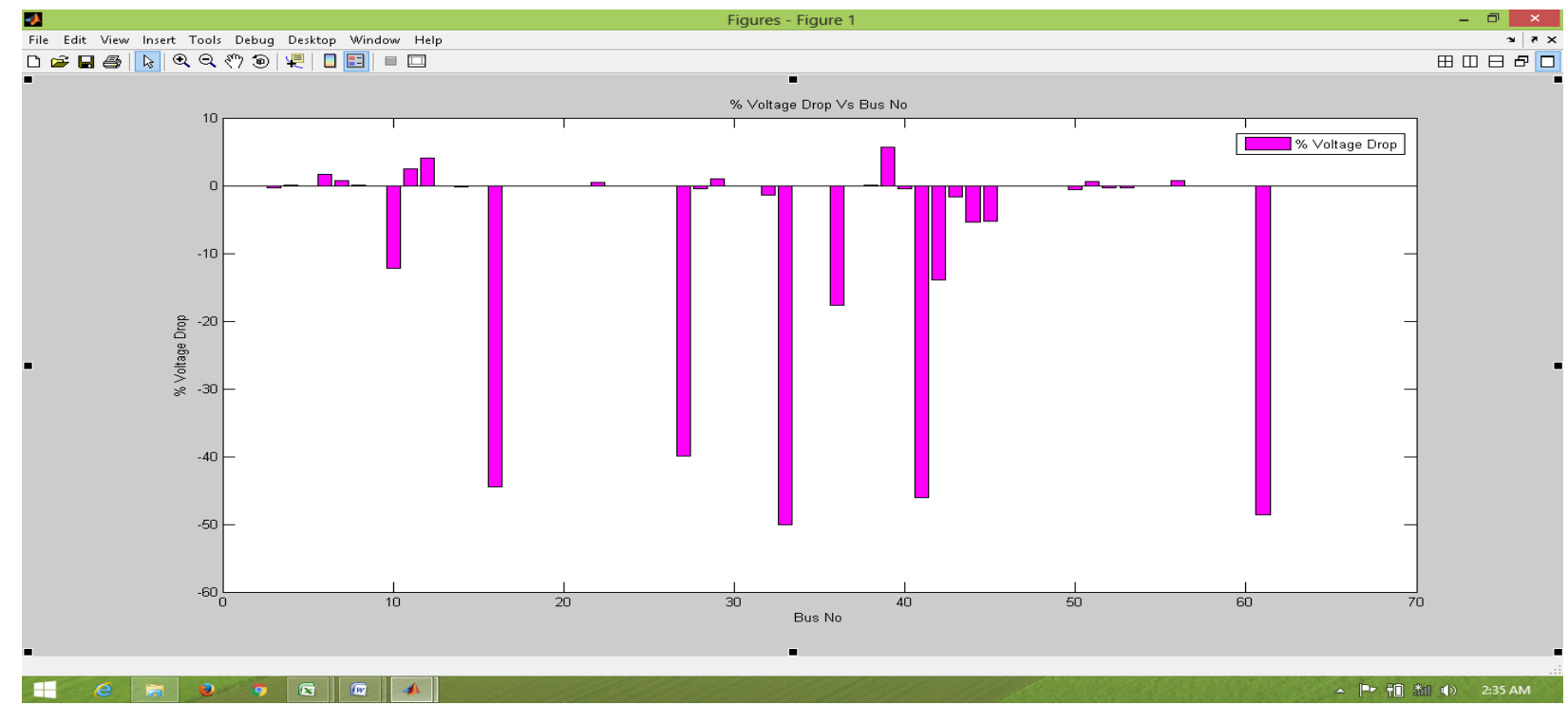

Figure 7: Percentage Voltage Drop Vs Bus No of Proposed Integrated 330kV Network

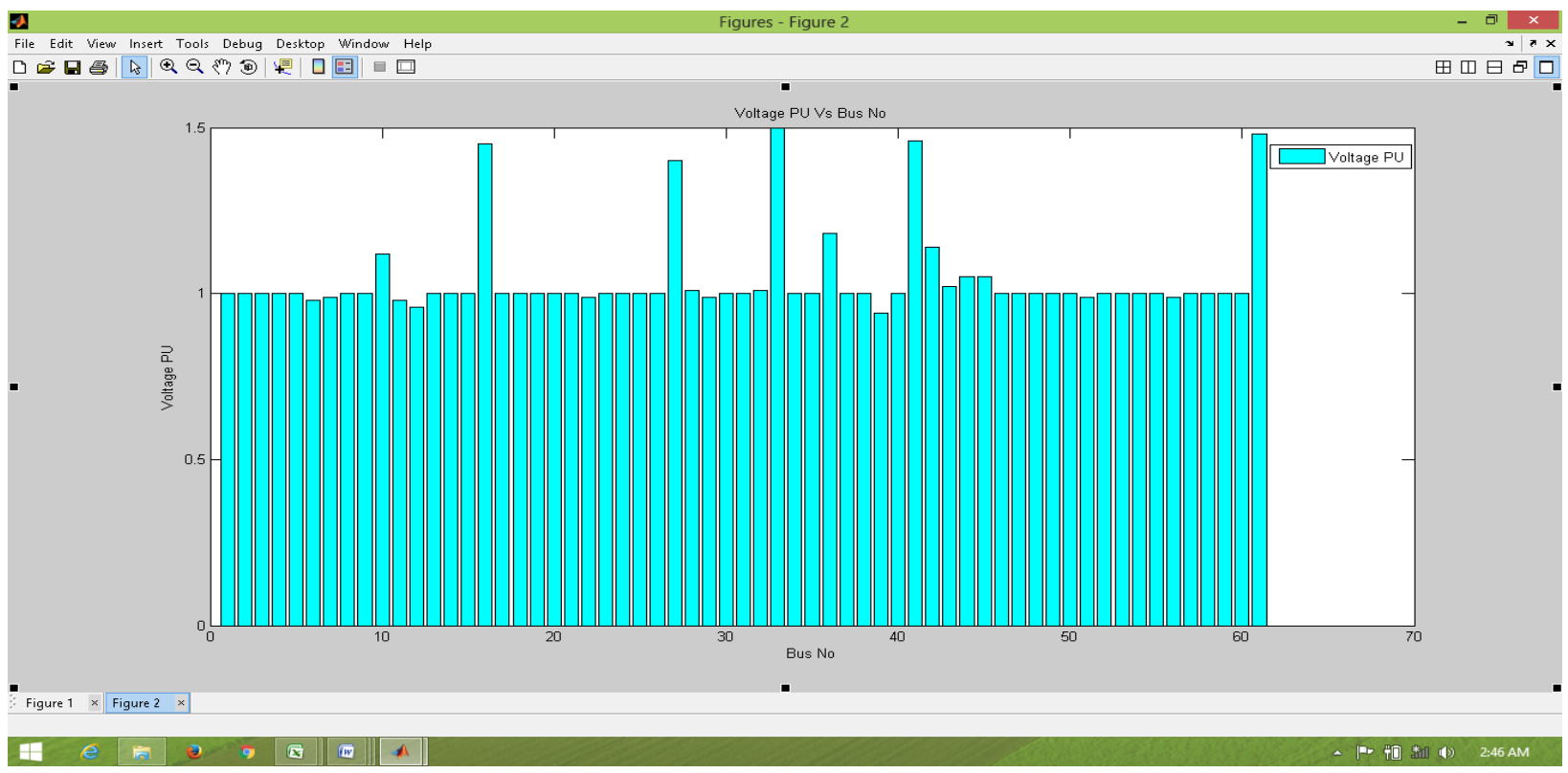

Figure 8: Voltage per unit Vs Bus No of Proposed Integrated 330KV Network

Similarly, plotting the graph of Voltage per unit Vs Bus No. of the Proposed Integrated 330KV network using MATLAB 7.5.0, figure 8 was obtained.

The real and reactive power losses in the network are 62.281MW and -3850.717MVar. The following transmission stations that were operating below the acceptable voltage limit of $\pm 5 \%$ were improved as follows; Gombe T.S from $218.91 \mathrm{KV}$ to $461.61 \mathrm{KV}$, Jos T.S from $263.05 \mathrm{KV}$ to $388.33 \mathrm{KV}$, Kumbotso T.S from $280.25 \mathrm{KV}$ to $311.29 \mathrm{KV}$ and Mando T.S from $308 \mathrm{KV}$ to $335.39 \mathrm{KV}$. Other buses in the network also have relative improvement on their bus voltages, thus resulting to improved voltage profile on the entire network. 
The summary of the results of the Existing 330KV network and the Proposed Integrated 330KV network using ETAP 12.6 Software is tabulated in table 3 .

Table 3: Summary of the result of existing and proposed 330KV network from ETAP 12.6

\begin{tabular}{|c|c|c|c|c|c|}
\hline Network & $\begin{array}{c}\text { Voltage } \\
\text { Profile } \\
\text { (PU) }\end{array}$ & $\begin{array}{c}\text { Active } \\
\text { Load } \\
\text { Demand } \\
(\mathrm{MW})\end{array}$ & $\begin{array}{c}\text { Reactive } \\
\text { Load } \\
\text { Demand } \\
\text { (Mvar) }\end{array}$ & $\begin{array}{c}\text { Losses } \\
\text { (Real) } \\
\text { MW }\end{array}$ & $\begin{array}{c}\text { Losses } \\
\text { (Reactive) } \\
\text { Mvar }\end{array}$ \\
\hline $\begin{array}{l}\text { Existing } \\
330 \mathrm{KV}\end{array}$ & $\begin{array}{c}0.66- \\
1.31\end{array}$ & 3232.42 & 337.91 & 49.72 & -1525.49 \\
\hline $\begin{array}{c}\text { Proposed } \\
\text { Integrated } \\
330 \mathrm{KV}\end{array}$ & $0.94-$ & 3311.11 & -1949.695 & 62.28 & -3850.72 \\
\hline
\end{tabular}

From the load flow analysis summarized in table 3, by the Rule of thumb, the average losses on each transmission line can be analyzed as follows;

For the existing $330 \mathrm{kV}$ network, 48 transmission lines exist and the losses in megawatt as indicated in ETAP 12.6 software is 49.72MW, hence the average loss in each of the transmission lines is therefore $1.04 \mathrm{MW}$ and the losses in MVar in the network is 1525.49MVar.

Similarly, for Proposed Integrated 330KV Network, the number of transmission line is 111, the losses in megawatt as indicated by ETAP 12.6 is $62.28 \mathrm{MW}$, hence the average loss in each transmission line is $0.56 \mathrm{MW}$ and the losses in MVar on the network is 3850.72MVar.

From the analysis, with the Proposed Integrated 330KV Network, the average loss on each transmission line has been reduced by $0.48 \mathrm{MW}$, which indicates $46 \%$ improvement on the Existing $330 \mathrm{kV}$ transmission network and the voltage profile on each bus of the entire network has also improved from $0.66 \mathrm{PU}-1.31 \mathrm{PU}$ to $0.94 \mathrm{PU}-1.50 \mathrm{PU}$.

\section{CONCLUSION}

With the integration of the proposed $330 \mathrm{KV}$ network, electricity supply in the national grid will be more stable, reliable and efficient as a result of the improved voltage profile on the buses and reduced transmission losses on the entire the network.

\section{REFERENCE}

Omoroguiwa E. \& Ike S. (2014). "Power flow control in the Nigeria 330KV integrated power network using unified power flow controller", International Journal of Engineering Innovation and Research, Vol.3, Issue 6, Pages 724 - 731, October. 
Onohaebi O.S. (2007). "Reduction of the high technical power losses associated with the Nigerian 330KV Transmission Network", International Journal of Electrical and Electronics Engineering, Vol 1(4), Pages 421-431.

Sambo A. S, Garba B., Zarma I. H., and Gaji M. M. (2012). "Electricity Generation and the present challenges in the Nigerian power sector", Energy commission of Nigeria.

Shaikh F. A, Ramanshu J., Mukesh K. and Nickey A. (2102). "New Techniques for the Prevention of Power System Collapse", International Journal of Electrical and Electronics Engineering, Vol. 1, Issue 3, Pages 123 - 129. 